\title{
Tip Trajectory Tracking for Multilink Flexible Manipulators Using Stable Inversion
}

\author{
Hongchao Zhao* and Degang Chen ${ }^{\dagger}$ \\ Iowa State University, Ames, Iowa 50011-3060
}

\begin{abstract}
The recently developed stable inversion theory for nonminimum phase nonlinear systems is applied to output tracking for multilink flexible robot manipulators. The stable inversion theory and a numerical solution to stable inverses are briefly reviewed. Forward dynamics of a two-link flexible manipulator with tip position as the output is then given using the assumed modes method. From that, an inverse model is derived and a two-point boundary value condition is set up. This condition guarantees that the inverse solution for a given desired tip trajectory will be stable regardless of the fact that a flexible manipulator is a nonminimum-phase system. The stable inverse is incorporated into an output tracking controller that uses only joint-angle feedback. Excellent tracking is achieved with no transient or steady-state errors and no internal vibration buildup.
\end{abstract}

\section{Introduction}

T HE most elementary task in robot control is to drive the end effector of a robot arm to follow a given desired trajectory. Precise positioning and appropriate speed control of the end effector along a given path are key requirements in many industrial applications such as arc welding, spray painting, pressure casting, tool machine serving, assembling, and thermal treatmentprocessing. All of these applications demand good designs on output trajectory tracking controllers for various robotic systems.

Robotics has been an active research area for the past few decades. ${ }^{1,2}$ The study on control of flexible robot manipulators was pioneered in Ref. 3, where a linear-quadratic optimal control approach was successfully applied to the end-effector tracking control of a one-link flexible robot arm in which the nonminimum-phase effect was first demonstrated.After that, many researchershave considered different approaches to the control of one-link flexible arms that are linear systems for small deflection. Among those, Siciliano and Book $^{4}$ used a singular perturbation approach to deal with the flexible modes. Bayo ${ }^{5}$ applied Fourier transform to obtain stable but noncausal control input. Kwon and Book's ${ }^{6}$ method is time domain and achieves the same effects as those of Bayo's. As for the nonlinear control of multilink flexible manipulators, Lucibello and Di Beneketto $^{7}$ applied the nonlinearregulation theory, ${ }^{8}$ and asymptotic tracking of periodic output trajectories was achieved. In a similar approachby De Luca et al., ${ }^{9}$ simulation results demonstrated asymptotic tracking of a finite trajectory with transient errors at both the beginning and the end of the maneuver.

This transient behavior can be precisely controlled by applying the classical inversion method that uses stabilizing feedback together with feedforward signals generated by an inverse system. Conditions for the invertibility of linear systems were developed by Brockett and Mesarovic, ${ }^{10}$ Silverman, ${ }^{11}$ and Sain and Massey. ${ }^{12}$ Inversion of nonlinear real-analytic systems was established by Hirschorn ${ }^{13}$ and Singh. ${ }^{14}$ All of these inversion algorithms produce causal inverses for a given desired output and a fixed initial condition, but unbounded control and state trajectories will be produced for nonminimum-phase systems. This fundamental difficulty has been noticed for a long time.

This paper, an extended version of Ref. 15, develops a new tracking control strategy for multilink flexible robot manipulators. The controller uses a feedforward signal generated by stable inversion ${ }^{16}$ and a stabilizing signal from a feedbackstabilizer. This design avoids the transient problem in the nonlinear regulation approach and the

Received Feb. 10, 1997; revision received July 20, 1997; accepted for publication Aug. 15, 1997. Copyright (C) 1997 by the American Institute of Aeronautics and Astronautics, Inc. All rights reserved.

${ }^{*}$ Ph.D. Candidate, Department of Electrical and Computer Engineering.

${ }^{\dagger}$ Assistant Professor, Department of Electrical and Computer Engineering. internal-dynamicsinstability problem in classicalinversion. As a result, remarkably accurate output tracking together with closed-loop stability will be achieved. The remainder of the paper is organized as follows. Section II briefly reviews the stable inversion theory and a numerical solution to stable inverses. Section III describes the forward dynamics of a two-link flexible manipulator and derives its stable inverse dynamics. In Sec. IV, conditions for applying stable inversion are verified and the effectiveness of stable inversion to output tracking for nonminimum-phase systems is demonstrated by simulation. A conclusion is given in Sec. V.

\section{Stable Inversion}

Consider multivariable nonlinear control systems of the form

$$
\begin{gathered}
\dot{x}=f(x)+G(x) u \\
y=h(x)
\end{gathered}
$$

where the system state $x$ is defined on an open neighborhood of the origin of $\mathbb{R}^{n}$ and the input $\boldsymbol{u} \in \mathbb{R}^{m}$ and the output $\boldsymbol{y} \in \mathbb{R}^{m}$. Smoothness of $\boldsymbol{f}(), G()$, and $\boldsymbol{h}$ () is assumed. Without loss of generality, we also assume that $\boldsymbol{f}(\boldsymbol{0})$ and $\boldsymbol{h}(\boldsymbol{0})=\mathbf{0}$. For such systems, a stable inversion problem is posed as follows. ${ }^{16}$

Given a smooth reference output trajectory $\boldsymbol{y}_{d}(t)$ with compact support, find a control input $\boldsymbol{u}_{d}(t)$ and a state trajectory $\boldsymbol{x}_{d}(t)$ such that 1) $\boldsymbol{u}_{d}(t)$ and $\boldsymbol{x}_{d}(t)$ satisfy the differential equation

$$
\dot{\boldsymbol{x}}_{d}(t)=\boldsymbol{f}\left(\boldsymbol{x}_{d}(t)\right)+G\left(\boldsymbol{x}_{d}(t)\right) \boldsymbol{u}_{d}(t)
$$

2) exact output tracking is achieved:

$$
\boldsymbol{h}\left(\boldsymbol{x}_{d}(t)\right)=\boldsymbol{y}_{d}(t)
$$

and 3) $\boldsymbol{u}_{d}(t)$ and $\boldsymbol{x}_{d}(t)$ are bounded and

$$
\boldsymbol{u}_{d}(t) \rightarrow \mathbf{0}, \quad \boldsymbol{x}_{d}(t) \rightarrow \mathbf{0} \quad \text { as } \quad t \rightarrow \pm \infty
$$

Here $\boldsymbol{u}_{d}(t)$ and $\boldsymbol{x}_{d}(t)$ are referred to as the stable inverse solution for a given reference output $\boldsymbol{y}_{d}(t)$. They are called stable inverses because of the boundedness and convergence provided by condition 3 . In addition, $\boldsymbol{x}_{d}(t)$ is called the desired state trajectory and $\boldsymbol{u}_{d}(t)$ the nominal control input.

\section{A. Two-Point Boundary Condition}

To find $\boldsymbol{x}_{d}(t)$ and $\boldsymbol{u}_{d}(t)$ for a given $\boldsymbol{y}_{d}(t)$, the following steps can be used. First, set $\boldsymbol{y}(t) \equiv \boldsymbol{y}_{d}(t)$ in the output equations (2). Second, differentiate each output equation until at least one input variable appears explicitly. This step will give us $m$ equations of the form

$$
y_{d_{i}}^{\left(r_{i}\right)}=\bar{a}_{i}(\boldsymbol{x})+\overline{\boldsymbol{b}}_{i}(\boldsymbol{x}) \boldsymbol{u}, \quad \forall i=1, \ldots, m
$$


where each $\overline{\boldsymbol{b}}_{i}(\boldsymbol{x})$ is a $1 \times m$ row vector. Next, we do a change of state variables. Let $\boldsymbol{\xi}$ contain all of the derivatives of the output variables:

$$
\boldsymbol{\xi} \stackrel{\text { def }}{=}\left[y_{1}, \dot{y}_{1}, \ldots, y_{1}^{\left(r_{1}-1\right)}, y_{2}, \ldots, y_{2}^{\left(r_{2}-1\right)}, \ldots, y_{m}^{\left(r_{m}-1\right)}\right]^{T}
$$

Choose $\boldsymbol{\eta}$ to contain any $(n-\operatorname{dim}(\boldsymbol{\xi}))$ variables that are linearly independent of $\boldsymbol{\xi}$ such that $\left[\boldsymbol{\xi}^{T}, \boldsymbol{\eta}^{T}\right]^{T}$ qualifies as a new set of state variables. Under the new variables

$$
\left[\begin{array}{l}
\xi \\
\eta
\end{array}\right] \stackrel{\text { def }}{=}\left[\begin{array}{l}
\phi_{1}(x) \\
\phi_{2}(x)
\end{array}\right] \stackrel{\text { def }}{=} \phi(x)
$$

the $m$ equations in Eq. (3) collectively become

$$
\boldsymbol{y}_{d}^{(r)}=\overline{\boldsymbol{a}}(\boldsymbol{\xi}, \boldsymbol{\eta})+\bar{B}(\boldsymbol{\xi}, \boldsymbol{\eta}) \boldsymbol{u}
$$

and differentiating $\boldsymbol{\eta}$ in Eq. (5) leads to

$$
\dot{\boldsymbol{\eta}}=\overline{\boldsymbol{c}}(\boldsymbol{\xi}, \boldsymbol{\eta})+\bar{D}(\boldsymbol{\xi}, \boldsymbol{\eta}) \boldsymbol{u}
$$

where

$$
\begin{aligned}
& \overline{\boldsymbol{c}}(\boldsymbol{\xi}, \boldsymbol{\eta})=\left.\frac{\partial \phi_{2}(\boldsymbol{x})}{\partial \boldsymbol{x}} \boldsymbol{f}(\boldsymbol{x})\right|_{\boldsymbol{x}=\phi^{-1}(\xi, \boldsymbol{\eta})} \\
& \bar{D}(\boldsymbol{\xi}, \boldsymbol{\eta})=\left.\frac{\partial \phi_{2}(\boldsymbol{x})}{\partial \boldsymbol{x}} G(\boldsymbol{x})\right|_{\boldsymbol{x}=\phi^{-1}(\xi, \boldsymbol{\eta})}
\end{aligned}
$$

Because we have set $\boldsymbol{y}(t) \equiv \boldsymbol{y}_{d}(t)$, we have

$$
\boldsymbol{\xi}=\boldsymbol{\xi}_{d} \stackrel{\text { def }}{=}\left[y_{d 1}, \dot{y}_{d 1}, \ldots, y_{d 1}^{\left(r_{1}-1\right)}, y_{d 2}, \ldots, y_{d 2}^{\left(r_{2}-1\right)}, \ldots, y_{d m}^{\left(r_{m}-1\right)}\right]^{T}
$$

Solving for $\boldsymbol{u}$ from Eq. (6), we obtain (assuming local invertibility ${ }^{16}$ )

$$
\boldsymbol{u}=\left[\bar{B}\left(\boldsymbol{\xi}_{d}, \boldsymbol{\eta}\right)\right]^{-1}\left[\boldsymbol{y}_{d}^{(r)}-\overline{\boldsymbol{a}}\left(\boldsymbol{\xi}_{d}, \boldsymbol{\eta}\right)\right]
$$

Substituting this into Eq. (7) leads to the so-called reference dynamics,

$$
\begin{aligned}
\dot{\boldsymbol{\eta}} & =\overline{\boldsymbol{c}}\left(\boldsymbol{\xi}_{d}, \boldsymbol{\eta}\right)+\bar{D}\left(\boldsymbol{\xi}_{d}, \boldsymbol{\eta}\right)\left[\bar{B}\left(\boldsymbol{\xi}_{d}, \boldsymbol{\eta}\right)\right]^{-1}\left[\boldsymbol{y}_{d}^{(r)}-\overline{\boldsymbol{a}}\left(\boldsymbol{\xi}_{d}, \boldsymbol{\eta}\right)\right] \\
& \stackrel{\text { def }}{=} P\left(\boldsymbol{y}_{d}^{(r)}, \boldsymbol{\xi}_{d}, \boldsymbol{\eta}\right)
\end{aligned}
$$

It is noticed that the $\boldsymbol{\eta}$ dynamics is the part of the system made unobservable from the output by the particular state feedback (10). Finally, if a bounded and convergent solution $\eta_{d}(t)$ can be obtained from Eq. (11), it can be used in Eq. (10) to obtain $\boldsymbol{u}_{d}(t)$ :

$$
\boldsymbol{u}_{d}=\left[\bar{B}\left(\boldsymbol{\xi}_{d}, \boldsymbol{\eta}_{d}\right)\right]^{-1}\left[\boldsymbol{y}_{d}^{(r)}(t)-\overline{\boldsymbol{a}}\left(\boldsymbol{\xi}_{d}, \boldsymbol{\eta}_{d}\right)\right]
$$

The inverse change of state variables by Eq. (5) then gives $\boldsymbol{x}_{d}(t)$ :

$$
\boldsymbol{x}_{d}=\phi^{-1}\left(\left[\boldsymbol{\xi}_{d}^{T}, \boldsymbol{\eta}_{d}^{T}\right]^{T}\right)
$$

Note that, when $\boldsymbol{y}_{d}(t) \equiv \mathbf{0}$ (and, consequently, $\left.\boldsymbol{\xi}_{d} \equiv \mathbf{0}\right)$, Eq. (11) becomes

$$
\dot{\boldsymbol{\eta}}=P(\mathbf{0}, \mathbf{0}, \boldsymbol{\eta})
$$

which is the so-called zero dynamics.

For $t$ outside $\left[t_{0}, t_{f}\right]$, the compact support of $\boldsymbol{y}_{d}(t)$, the reference dynamics (11) becomes the autonomous zero dynamics (14). Assume that $\boldsymbol{\eta}=\mathbf{0}$ is a hyperbolic equilibrium point of the zero dynamics. It has been shown that any bounded and convergent solution $\boldsymbol{\eta}_{d}(t)$ of Eq. (11) must satisfy the following two-point boundary value (TPBV) problem ${ }^{16}$ :

$$
\dot{\boldsymbol{\eta}}_{d}=P\left(\boldsymbol{y}_{d}^{(r)}, \boldsymbol{\xi}_{d}, \boldsymbol{\eta}_{d}\right)
$$

subject to

$$
\boldsymbol{\eta}_{d}\left(t_{0}\right) \in \mathbb{W}^{u}, \quad \boldsymbol{\eta}_{d}\left(t_{f}\right) \in \mathbb{W}^{s}
$$

where $\mathbb{W}^{u}$ is the invariant unstable submanifold and $\mathbb{W}^{s}$ the stable submanifold of the zero dynamics (14). That is, before $t_{0}$ the reference dynamics remains on the unstable submanifold, whereas after $t_{f}$ it remains on the stable submanifold. In the linear case, such submanifolds correspond to the stable and unstable eigenspaces. Both submanifolds of the zero dynamics are, respectively, tangent to their corresponding eigenspaces of the linearized zero dynamics near $\boldsymbol{\eta}=\mathbf{0}$.

\section{B. Iterative Solution to the TPBV Problem}

The key to obtaining $\boldsymbol{x}_{d}(t)$ and $\boldsymbol{u}_{d}(t)$ is to solve for a bounded and convergent $\boldsymbol{\eta}_{d}(t)$ from Eqs. (15) and (16), the TPBV problem. An iterative linearization approach to such a solution is described as follows.

In each iteration, the differential equation (15) is linearized along the solution obtained from the preceding iteration to yield Eq. (17). The stable and unstable eigenspaces, $\mathbb{E}^{s}$ and $\mathbb{E}^{u}$, respectively, of the zero dynamics corresponding to Eq. (17), are used as the boundary conditions instead of $\mathbb{W}^{s}$ and $\mathbb{W}^{u}$. Thus, yielding a linear timevarying TPBV problem at this iteration,

$$
\dot{\eta}_{d}=A(t) \eta_{d}+B(t)
$$

subject to

$$
\boldsymbol{\eta}_{d}\left(t_{0}\right) \in \mathbb{E}^{u}, \quad \boldsymbol{\eta}_{d}\left(t_{f}\right) \in \mathbb{E}^{s}
$$

The boundary condition (18) can be characterizedby two equality conditions. To do this, let matrix $X_{s}\left(t_{0}\right)\left(Y_{s}\left(t_{0}\right)\right)$ contain the real right (left) eigenvectors and the generalized eigenvectors of $A\left(t_{0}\right)$ associated with eigenvalues having negative real parts and $X_{u}\left(t_{0}\right)$ $\left(Y_{u}\left(t_{0}\right)\right)$ contain those associated with eigenvalues having positive real parts. Then, we have

$$
\left[\begin{array}{l}
Y_{s}\left(t_{0}\right) \\
Y_{u}\left(t_{0}\right)
\end{array}\right] A\left(t_{0}\right)\left[X_{s}\left(t_{0}\right) \quad X_{u}\left(t_{0}\right)\right]=\left[\begin{array}{cc}
J_{s}\left(t_{0}\right) & O \\
O & J_{u}\left(t_{0}\right)
\end{array}\right]
$$

where $J_{s}\left(t_{0}\right)$ and $J_{u}\left(t_{0}\right)$ are the corresponding real Jordan canonical forms of the stable and unstable subspaces, respectively. In particular, from Eq. (19) we have

$$
Y_{s}\left(t_{0}\right) A\left(t_{0}\right) X_{u}\left(t_{0}\right)=O
$$

On the other hand, the condition $\boldsymbol{\eta}_{d}\left(t_{0}\right) \in \mathbb{E}^{u}$ can be characterized by $\boldsymbol{\eta}_{d}\left(t_{0}\right)$ expressed as a linear combination of unstable right eigenvectors and generalized eigenvectors. That is,

$$
\boldsymbol{\eta}_{d}\left(t_{0}\right)=X_{u}\left(t_{0}\right) \boldsymbol{z}_{u}
$$

for some vector $z_{u}$. Combining this with Eq. (20) yields an equivalent equality condition for $\boldsymbol{\eta}_{d}\left(t_{0}\right) \in \mathbb{E}^{u}$

$$
C_{s} \boldsymbol{\eta}_{d}\left(t_{0}\right)=\mathbf{0}
$$

where

$$
C_{s} \stackrel{\text { def }}{=} Y_{s}\left(t_{0}\right) A\left(t_{0}\right)
$$

Similar derivation at $t_{f}$ can be made to replace $\boldsymbol{\eta}_{d}\left(t_{f}\right) \in \mathbb{E}^{s}$ by

$$
C_{u} \boldsymbol{\eta}_{d}\left(t_{f}\right)=\mathbf{0}
$$

where

$$
C_{u} \stackrel{\text { def }}{=} Y_{u}\left(t_{f}\right) A\left(t_{f}\right)
$$

The linear problem [Eqs. (17) and (22-25)] is then solved, and the solution is taken to be the new approximation of the current iteration. The iteration continues until the solutions in the adjacent two iterations are satisfactorily close to each other. Solving the boundary value problem in Eqs. (17) and (22-25) is done following a technique from linear-quadratic optimal control and is carried out in the following steps.

First, apply a change of state variable,

$$
\zeta \stackrel{\text { def }}{=}\left[\begin{array}{l}
\zeta_{1} \\
\zeta_{2}
\end{array}\right] \stackrel{\text { def }}{=}\left[\begin{array}{l}
C_{s} \\
C_{u}
\end{array}\right] \boldsymbol{\eta}_{d}
$$


Because $C_{s} \boldsymbol{\eta}_{d}=0$ in Eq. (22) characterizes the unstable eigenspace of the zero dynamics, therefore, $\zeta_{1}=C_{s} \boldsymbol{\eta}_{d}$ is, roughly speaking, the stable part of $\boldsymbol{\eta}_{d}$. Similarly, $\zeta_{2}=C_{u} \boldsymbol{\eta}_{d}$ is the unstable part. The inverse transformation is given by

$$
\boldsymbol{\eta}_{d}=\left[\begin{array}{l}
C_{s} \\
C_{u}
\end{array}\right]^{-1} \zeta \stackrel{\text { def }}{=}\left[\begin{array}{ll}
T_{s} & T_{u}
\end{array}\right]\left[\begin{array}{l}
\zeta_{1} \\
\zeta_{2}
\end{array}\right]
$$

Differentiating $\zeta_{1}$ and $\zeta_{2}$ using Eqs. (17), (26), and (27), we get

$$
\begin{aligned}
& \dot{\zeta}_{1}=A_{11}(t) \zeta_{1}+A_{12}(t) \zeta_{2}+B_{1}(t) \\
& \dot{\zeta}_{2}=A_{21}(t) \zeta_{1}+A_{22}(t) \zeta_{2}+B_{2}(t)
\end{aligned}
$$

and the boundary conditions in Eqs. (22-25) become $\zeta_{1}\left(t_{0}\right)$ and $\boldsymbol{\zeta}_{2}\left(t_{f}\right)=\mathbf{0}$. Note that $\boldsymbol{\zeta}_{1}\left(t_{0}\right)=\mathbf{0}$ and Eq. (28) form an initial value problem, whereas $\zeta_{2}\left(t_{f}\right)=\mathbf{0}$ and Eq. (29) form a final value problem. However, these two problems are coupled.

Second, decouple the $\boldsymbol{\zeta}_{1}$ and $\boldsymbol{\zeta}_{2}$ dynamics. Because $\boldsymbol{\zeta}_{1}$ and $\boldsymbol{\zeta}_{2}$ satisfy a pair of linear differential equations, their solutions are also linearly related. That is,

$$
\zeta_{2}(t)=S(t) \zeta_{1}(t)+v(t)
$$

for some functions $S(t)$ and $v(t)$ with suitable final value conditions

$$
S\left(t_{f}\right)=O \quad \text { and } \quad \boldsymbol{v}\left(t_{f}\right)=\mathbf{0}
$$

Differentiating both sides of Eq. (30) yields

$$
\dot{\zeta}_{2}(t)=\dot{S}(t) \zeta_{1}(t)+S(t) \dot{\boldsymbol{\zeta}}_{1}(t)+\dot{\boldsymbol{v}}(t)
$$

Substituting the values of $\dot{\zeta}_{1}$ and $\dot{\zeta}_{2}$ from Eqs. (28) and (29) and comparing the coefficients of $\zeta_{1}(t)$ leads to

$$
\begin{gathered}
\dot{S}(t)=A_{21}(t)+A_{22}(t) S(t)-S(t) A_{11}(t)-S(t) A_{12}(t) S(t) \\
\dot{\boldsymbol{v}}(t)=\left[A_{22}(t)-S(t) A_{12}(t)\right] \boldsymbol{v}(t)+\left[B_{2}(t)-S(t) B_{1}(t)\right]
\end{gathered}
$$

with final conditions specified in Eq. (31).

Third, backward and forward integrate. Because Eq. (32) contains only known functions except $S(t)$, it can be integrated backward in time to get $S(t)$. Once this is done, Eq. (33) can also be integrated backward in time to solve for $\boldsymbol{v}(t)$. With $S(t)$ and $\boldsymbol{v}(t)$ as known functions, Eq. (28) can be rewritten as

$$
\dot{\boldsymbol{\zeta}}_{1}=\left[A_{11}(t)+A_{12}(t) S(t)\right] \boldsymbol{\zeta}_{1}+B_{1}(t)+A_{12}(t) \boldsymbol{v}(t)
$$

and it can be integrated forward in time with $\boldsymbol{\zeta}_{1}\left(t_{0}\right)=\mathbf{0}$ to obtain $\zeta_{1}(t)$. With these, the algebraic equation (30) can be used to obtain $\zeta_{2}(t)$.

The final step is to use the inverse transformation in Eq. (27) to obtain $\eta_{d}(t)$ that will be the solution of the current iteration.

Even though all stable inversion results are local and we have chosen a local linearization approach to construct stable inverses, the stable inverse solution can be, but does not have to be, always locally constructed.

\section{Forward and Inverse Dynamics}

Consider a two-link flexible robot manipulator shown in Fig. 1. Both joints of the links are considered to be revolute, and input torque is applied at these joints. Each link is assumed to be slender such that the Euler-Bernoulli beam assumption is valid. Horizontal planar maneuver is assumed so that we neglect out-of-plane deflection of both links.

\section{A. Forward System Dynamics}

For rigid-body mechanical systems and single-link flexible robot arms, dynamic modeling can be easily handled by applying Lagrange's principle. ${ }^{17}$ However, the dynamics of multilink articulated flexible structures is more complicated. Some researchershave used a finite element method to numerically construct the dynamic equations. ${ }^{18}$ Others have used the assumed modes approach. ${ }^{19,20}$ In this paper, the assumed modes method is also used to parameterize

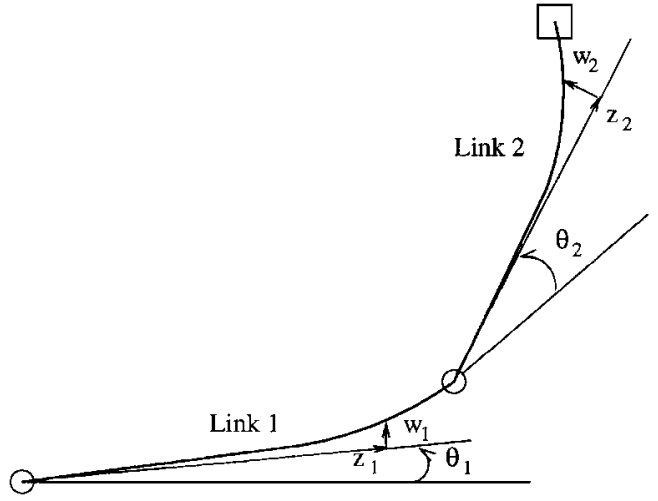

Fig. 1 Two-link flexible robot arm.

the continuous deformation of both flexible links. The following discussion on forward dynamics is included for the sake of fixing notations. Detailed derivation of the forward dynamics is omitted due to the availability of the relevant references.

Let $z_{i}$ measure the distance of a point at link $i$ in the direction of the undeformed link position and $w_{i}$ the deformation for the $i$ th link for $i=1$ and 2. Let $\sigma_{i j}\left(z_{i}\right)$ be the $j$ th admissible function for the $i$ th link and $q_{i j}(t)$ the corresponding generalized coordinates. Then the distributed deflection of the $i$ th link, $w_{i}$, is approximated by

$$
w_{i}\left(z_{i}, t\right)=\sum_{j=1}^{n_{i}} \sigma_{i j}\left(z_{i}\right) q_{i j}(t), \quad \forall_{i}=1,2
$$

The admissible functions are chosen to be the ones for the clampedfree beams ${ }^{21}$ and two flexible modes are assigned to each link, $n_{i}=2$. One simple choice of the admissible functions is of the form

$$
\sigma_{i j}\left(z_{i}\right)=\left[z_{i} / l_{i}\right]^{j+1}, \quad \forall j=1,2, \quad \forall i=1,2
$$

where $l_{i}$ is the length of link $i$ for $i=1$ and 2 . The geometric boundary conditions are all satisfied because the polynomials in Eq. (36) always have $\sigma_{i j}(0)=\sigma_{i j}^{\prime}(0)=0$, where $\sigma_{i j}^{\prime}()$ denotes the derivative of $\sigma_{i j}\left(\right.$ ) with respect to its spatial variable $z_{i}$.

Denote the system's generalized coordinates

$$
\psi \stackrel{\text { def }}{=}\left[\theta_{1}, \theta_{2}, q_{11}, q_{12}, q_{21}, q_{22}\right]^{T}
$$

which consist of the rigid modes $\boldsymbol{\theta}$ and the flexible modes $\boldsymbol{q}$ for both links:

$$
\boldsymbol{\theta} \stackrel{\text { def }}{=}\left[\theta_{1}, \theta_{2}\right]^{T}, \quad \boldsymbol{q} \stackrel{\text { def }}{=}\left[q_{11}, q_{12}, q_{21}, q_{22}\right]^{T}
$$

By Lagrange's method, the equations of motion can be written as follows:

$$
M(\psi) \ddot{\psi}+H(\psi, \dot{\psi})+C \dot{\psi}+K \boldsymbol{\psi}=B_{u} \boldsymbol{u}
$$

where $\boldsymbol{u}=\left[u_{1}, u_{2}\right]^{T}$ is the vector of joint torque and $B_{u}=\left[I_{2 \times 2}\right.$, $\left.\mathrm{O}_{4 \times 2}\right]^{T}$ is the torque distribution matrix. $K$ is the stiffness matrix

$$
K=\left[\begin{array}{cc}
O_{2 \times 2} & O_{2 \times 4} \\
O_{4 \times 2} & M_{3}
\end{array}\right]
$$

The damping matrix $C$ is taken to be proportional to the stiffness matrix $K$ by a damping ratio $\alpha$ :

$$
C=\left[\begin{array}{cc}
O_{2 \times 2} & O_{2 \times 4} \\
O_{4 \times 2} & M_{2}
\end{array}\right]
$$

where $M_{2}=\alpha M_{3} . M(\psi)$ is the $6 \times 6$ positive definite symmetric inertia matrix, and $H(\psi, \dot{\psi})$ the part containing centrifugal and Coriolis terms.

There are many ways to choose the system output. Depending on which point along the links is selected as the output, the whole system can be either minimum phase or nonminimum phase. If the 
output is selected to be the joint angles, i.e., the sensors and actuators are collocated, the system is known to be minimum phase. A more meaningful choice of the output is the tip position, and this choice renders the system nonminimum phase. We choose

$$
\boldsymbol{y} \stackrel{\text { def }}{=}\left[y_{1}, y_{2}\right]^{T}=\boldsymbol{\theta}+\left[\arctan \left(\frac{w_{1}\left(l_{1}, t\right)}{l_{1}}\right), \arctan \left(\frac{w_{2}\left(l_{2}, t\right)}{l_{2}}\right)\right]^{T}
$$

When elastic deformation of the first link is small, the output variables are approximately the tip angular positions of the links. It can be seen that both output components chosen are practically measurable. For small elastic deformation,

$$
\arctan \left(\frac{w_{i}\left(l_{i}, t\right)}{l_{i}}\right) \approx \frac{w_{i}\left(l_{i}, t\right)}{l_{i}}
$$

By substitutingEqs. (35) and (42) into Eq. (41), we obtain the output equation

$$
\boldsymbol{y}=D \psi
$$

where $D=\left[D_{1}, D_{2}\right]$ with $D_{1}=I_{2 \times 2}$ and

$$
D_{2}=\left[\begin{array}{cccc}
l_{1}^{-1} \sigma_{11}\left(l_{1}\right) & l_{1}^{-1} \sigma_{12}\left(l_{1}\right) & 0 & 0 \\
0 & 0 & l_{2}^{-1} \sigma_{21}\left(l_{2}\right) & l_{2}^{-1} \sigma_{22}\left(l_{2}\right)
\end{array}\right]
$$

Equation (38) together with Eq. (43) constitutes the forward dynamics of the two-link flexible robot manipulator system.

\section{B. Inverse Dynamics}

Inverse dynamics usually consists of reference dynamics (11) and an output equation (10). For a flexible manipulator system, the inverse dynamics can be simply derived as follows.

Rewrite the forward dynamics (38) and (43) as follows:

$$
\begin{gathered}
M_{11}(\psi) \ddot{\boldsymbol{\theta}}+M_{12}(\psi) \ddot{\boldsymbol{q}}+H_{1}(\boldsymbol{\psi}, \dot{\boldsymbol{\psi}})=\boldsymbol{u} \\
M_{21}(\boldsymbol{\psi}) \ddot{\boldsymbol{\theta}}+M_{22}(\psi) \ddot{\boldsymbol{q}}+H_{2}(\boldsymbol{\psi}, \dot{\psi})+M_{2} \dot{\boldsymbol{q}}+M_{3} \boldsymbol{q}=\mathbf{0} \\
\boldsymbol{y}=\boldsymbol{\theta}+D_{2} \boldsymbol{q}
\end{gathered}
$$

where $D_{2}$ is defined in Eq. (44) and $M_{2}$ and $M_{3}$ are defined in Eqs. (40) and (39), respectively. From Eq. (47), we have

$$
\boldsymbol{\theta}=\boldsymbol{y}-D_{2} \boldsymbol{q}
$$

Let $\boldsymbol{y}_{d}$ be the reference output trajectory. Set $\boldsymbol{y} \equiv \boldsymbol{y}_{d}$. Substituting Eq. (48) into Eq. (46), we obtain a dynamic equation governing the flexible coordinates $\boldsymbol{q}$ :

$$
M_{1}\left(\boldsymbol{y}_{d}, \boldsymbol{q}\right) \ddot{\boldsymbol{q}}+M_{2} \dot{\boldsymbol{q}}+M_{3} \boldsymbol{q}+H_{2}\left(\boldsymbol{y}_{d}, \dot{\boldsymbol{y}}_{d}, \boldsymbol{q}, \dot{\boldsymbol{q}}\right)=M_{4}\left(\boldsymbol{y}_{d}, \boldsymbol{q}\right) \ddot{\boldsymbol{y}}_{d}
$$

where

$$
\begin{gathered}
M_{1}\left(\boldsymbol{y}_{d}, \boldsymbol{q}\right)=M_{22}\left(\boldsymbol{y}_{d}, \boldsymbol{q}\right)-M_{21}\left(\boldsymbol{y}_{d}, \boldsymbol{q}\right) D_{2} \\
M_{4}\left(\boldsymbol{y}_{d}, \boldsymbol{q}\right)=-M_{21}\left(\boldsymbol{y}_{d}, \boldsymbol{q}\right)
\end{gathered}
$$

Equation (49) is the reference dynamics equation (11) in secondorder form.

The equivalence of the general equation (10) for the flexible manipulator can be easily obtained from Eq. (45) with a substitution of Eq. (48) and $\boldsymbol{y} \equiv \boldsymbol{y}_{d}$ :

$$
\begin{aligned}
\boldsymbol{u} & =\left[M_{12}\left(\boldsymbol{y}_{d}, \boldsymbol{q}\right)-M_{11}\left(\boldsymbol{y}_{d}, \boldsymbol{q}\right) D_{2}\right] \ddot{\boldsymbol{q}} \\
& +\left[M_{11}\left(\boldsymbol{y}_{d}, \boldsymbol{q}\right) \ddot{\boldsymbol{y}}_{d}+H_{1}\left(\boldsymbol{y}_{d}, \dot{\boldsymbol{y}}_{d}, \boldsymbol{q}, \dot{\boldsymbol{q}}\right)\right]
\end{aligned}
$$

Equations (49) and (50) characterize the inverse dynamics of the two-link flexible manipulator system.

\section{Linear TPBV Problem for Flexible Manipulators}

To ensure a stable solution from the inverse dynamics, a two-point boundary condition (16) needs to be imposed on the flexible mode $q$. However, instead of Eq. (16), we directly derive the linear TPBV problem (17) and (18) for our flexible manipulators, based on which the iterative procedure described in Sec. II.B can be carried out to find stable inverses.

First, we need to find the linearized equation for the reference dynamics (49). A notation for convenience is in order. Let $\mathcal{M}(\boldsymbol{x})$ be a $k_{m} \times l_{m}$ matrix function of $\boldsymbol{x} \in \mathbb{R}^{n}$ and $\overline{\boldsymbol{x}} \in \mathbb{R}^{n}$ be a column vector. The derivative of $\mathcal{M}$ at a point $\boldsymbol{x}_{0}$ in the direction of $\overline{\boldsymbol{x}}$ is defined as

$$
\left.D_{x}^{x_{0}} \mathcal{M} \bar{x} \stackrel{\text { def }}{=} \sum_{i=1}^{n} \frac{\partial \mathcal{M}}{\partial x_{i}}\right|_{x=x_{0}} \bar{x}_{i}
$$

Using this notation and neglecting higher-orderterms, the first term $M_{1} \ddot{q}$ in the reference dynamics equation (49) can be linearized as

$$
\begin{aligned}
M_{1} \ddot{\boldsymbol{q}} & \approx\left[M_{1}^{0}+D_{q}^{0} M_{1}\left[\boldsymbol{q}-\boldsymbol{q}_{0}\right]\right]\left[\ddot{\boldsymbol{q}}_{0}+\left[\ddot{\boldsymbol{q}}-\ddot{\boldsymbol{q}}_{0}\right]\right] \\
& \approx M_{1}^{0} \ddot{\boldsymbol{q}}+\left[D_{q}^{0} M_{1} \boldsymbol{q}\right] \ddot{\boldsymbol{q}}_{0}-\left[D_{q}^{0} M_{1} \boldsymbol{q}_{0}\right] \ddot{\boldsymbol{q}}_{0}
\end{aligned}
$$

where the superscript 0 stands for evaluation along $\boldsymbol{q}_{0}$ and/or $\dot{\boldsymbol{q}}_{0}$ (solution of the preceding iteration) no matter which is applicable. Because it can be easily verified that

$$
\left[D_{x} M \overline{\boldsymbol{x}}\right] \tilde{\boldsymbol{x}}=\left[D_{x} M \tilde{\boldsymbol{x}}\right] \overline{\boldsymbol{x}}
$$

where $\tilde{\boldsymbol{x}} \in \mathbb{R}^{n}$, we obtain

$$
M_{1} \ddot{\boldsymbol{q}} \approx M_{1}^{0} \ddot{\boldsymbol{q}}+\left[D_{q}^{0} M_{1} \ddot{\boldsymbol{q}}_{0}\right] \boldsymbol{q}-\left[D_{q}^{0} M_{1} \ddot{\boldsymbol{q}}_{0}\right] \boldsymbol{q}_{0}
$$

Both $M_{2}$ and $M_{3}$ are constantmatrices. For the term $H_{2}\left(\boldsymbol{y}_{d}, \dot{y}_{d}, \boldsymbol{q}, \dot{\boldsymbol{q}}\right)$, we have

$$
\begin{aligned}
H_{2} & \approx H_{2}^{0}+D_{q}^{0} H_{2}\left[\boldsymbol{q}-\boldsymbol{q}_{0}\right]+D_{\dot{q}}^{0} H_{2}\left[\dot{\boldsymbol{q}}-\dot{\boldsymbol{q}}_{0}\right] \\
& =H_{2}^{0}-D_{q}^{0} H_{2} \boldsymbol{q}_{0}-D_{\dot{q}}^{0} H_{2} \dot{\boldsymbol{q}}_{0}+D_{q}^{0} H_{2} \boldsymbol{q}+D_{\dot{q}}^{0} H_{2} \dot{\boldsymbol{q}}
\end{aligned}
$$

Similar to the derivation for the first term $M_{1} \ddot{\boldsymbol{q}}$, we can get the linearized form of $M_{4} \ddot{y}_{d}$ as

$$
M_{4} \ddot{\boldsymbol{y}}_{d} \approx M_{4}^{0} \ddot{\boldsymbol{y}}_{d}-\left[D_{q}^{0} M_{4} \ddot{\boldsymbol{y}}_{d}\right] \boldsymbol{q}_{0}+\left[D_{q}^{0} M_{4} \ddot{\boldsymbol{y}}_{d}\right] \boldsymbol{q}
$$

Thus, combining Eqs. (54-56), the linearized inverse dynamics can be expressed as

$$
L_{1} \ddot{\boldsymbol{q}}+L_{2} \dot{\boldsymbol{q}}+L_{3} \boldsymbol{q}=L_{4}
$$

where

$$
\begin{gathered}
L_{1}=M_{1}^{0}, \quad L_{2}=M_{2}+D_{\dot{q}}^{0} H_{2} \\
L_{3}=D_{q}^{0} M_{1} \ddot{\boldsymbol{q}}_{0}+M_{3}+D_{q}^{0} H_{2}-D_{q}^{0} M_{4} \ddot{\boldsymbol{y}}_{d} \\
L_{4}=M_{4}^{0} \ddot{\boldsymbol{y}}_{d}-\left[D_{q}^{0} M_{4} \ddot{\boldsymbol{y}}_{d}\right] \boldsymbol{q}_{0}+\left[D_{q}^{0} M_{1} \ddot{\boldsymbol{q}}_{0}\right] \boldsymbol{q}_{0} \\
+D_{q}^{0} H_{2} \boldsymbol{q}_{0}+D_{\dot{q}}^{0} H_{2} \dot{\boldsymbol{q}}_{0}-H_{2}^{0}
\end{gathered}
$$

Let $\boldsymbol{\eta}=\left[\boldsymbol{q}^{T}, \dot{\boldsymbol{q}}^{T}\right]^{T}$ and

$$
A(t)=\left[\begin{array}{cc}
0 & I \\
-L_{1}^{-1} L_{3} & -L_{1}^{-1} L_{2}
\end{array}\right], \quad B(t)=\left[\begin{array}{c}
0 \\
L_{1}^{-1} L_{4}
\end{array}\right]
$$

Then Eq. (57) is the same as Eq. (17) in second-orderform.

Second, the linear boundary conditions (22-25) are to be derived. Instead of updating the transformation matrices $C_{s}$ and $C_{u}$ at each iteration, in this simulation we compute one $C_{s}$ and one $C_{u}$ for all iterations. The matrix $A(t)$ in Eq.(58) is evaluated at $\boldsymbol{q}_{0}=\mathbf{0}$ and $\boldsymbol{y}_{d}=[0,90]^{T} \mathrm{deg}$. It is found later in the simulation by computing the eigenvalues of $A\left(t_{0}\right)$ and $A\left(t_{f}\right)$ that, at both $t_{0}$ and $t_{f}$, the zero dynamics has five stable eigenvalues and three unstable ones. Thus, following the procedure in Sec. II.B, the transformation matrices $C_{s}$ and $C_{u}$ would be of dimension $5 \times 8$ and $3 \times 8$, respectively. 


\section{Digital Simulation Study}

In this simulation study, we demonstrate the effectiveness of our proposed tracking control design using stable inversion. First, a simulation setup is presented. Then, some conditions are verified to ensure the applicability of stable inversion. This is followed by a tracking controllerdesign. Finally, some simulation results are presented.

\section{A. Simulation Setup}

Table 1 lists key parameters of the two-link flexible arm model used in this study. The two links of the manipulator are assumed to have the same structural damping with $\alpha=0.01$. and $e$ denote their length, mass, and product of area moment of inertia and Young's modulus, respectively. Also let $m_{e}$ and $h$ denote the endpoint mass and hub inertia of the second link, respectively.

Zero dynamics is first obtained from the reference dynamics (49) by setting $\boldsymbol{y}_{d}=[0,90]^{T}$ deg and derivatives of $\boldsymbol{y}_{d}$ of all orders to zero (an equilibrium point). Then, a standard linearization on the obtained zero dynamics yields linearized zero dynamics as follows:

$$
A_{1} \ddot{\boldsymbol{q}}+A_{2} \dot{\boldsymbol{q}}+A_{3} \boldsymbol{q}=\mathbf{0}
$$

where

$$
A_{1}=\left[\begin{array}{cccc}
\frac{37}{60} m+2 m_{e}+2\left(h / l^{2}\right) & \frac{5}{4} m+4 m_{e}+4\left(h / l^{2}\right) & -\frac{1}{6} m-2\left(h / l^{2}\right) & -\frac{4}{15} m-2\left(h / l^{2}\right) \\
\frac{29}{30} m+3 m_{e}+3\left(h / l^{2}\right) & \frac{68}{35} m+6 m_{e}+6\left(h / l^{2}\right) & -\frac{1}{4} m-3\left(h / l^{2}\right) & -\frac{2}{5} m-3\left(h / l^{2}\right) \\
\frac{1}{4} m+m_{e} & \frac{1}{2} m+2 m_{e} & -\frac{1}{20} m & -\frac{1}{12} m \\
\frac{1}{5} m+m_{e} & \frac{2}{5} m+2 m_{e} & -\frac{1}{30} m & -\frac{2}{35} m
\end{array}\right]
$$

The reference trajectory is selected following considerations given by Bayo and Paden. ${ }^{22}$ First, the acceleration profile should not have exceedingly high-frequency components. The reason is that, if the acceleration changes too rapidly, then the calculated torque profile will contain high peak impulse, which may excite the natural frequencies of the flexible manipulators. Second, the maximum acceleration limit should be chosen so as not to saturate the actuator. With these considerations, we have chosen the reference tip trajectory for link two as shown in Fig. 2, in which the acceleration profile is a sinusoidal function. A similar reference trajectory profile has been chosen for link one.

\section{B. Verification of System Properties}

To apply the stable inversion approach, two conditions need to be verified ${ }^{16}$ : the system should have a locally well-defined relative degree and its zero dynamics should have a hyperbolic equilibrium point at the origin.

Before we verify these, we first show the nonminimum-phase property of the system. Otherwise, the output trajectory tracking can be accomplished using the classical inversion approach. ${ }^{13}$ To make notationssimple, we assume that the two links are identical.Let $l, m$,

Table 1 Properties of the robot arm

\begin{tabular}{lcc}
\hline \hline Property & Link one & Link two \\
\hline$l, \mathrm{~m}$ & 1.0000 & 1.0000 \\
$\rho, \mathrm{kg} / \mathrm{m}^{2}$ & 0.3000 & 0.1000 \\
$e, \mathrm{~N} / \mathrm{m}^{2}$ & 3.9375 & 0.4375 \\
$m_{e}, \mathrm{~kg}$ & 0.1500 & 0.1000 \\
$h, \mathrm{~kg} / \mathrm{m}^{2}$ & 0.2000 & 0.0670 \\
\hline \hline
\end{tabular}
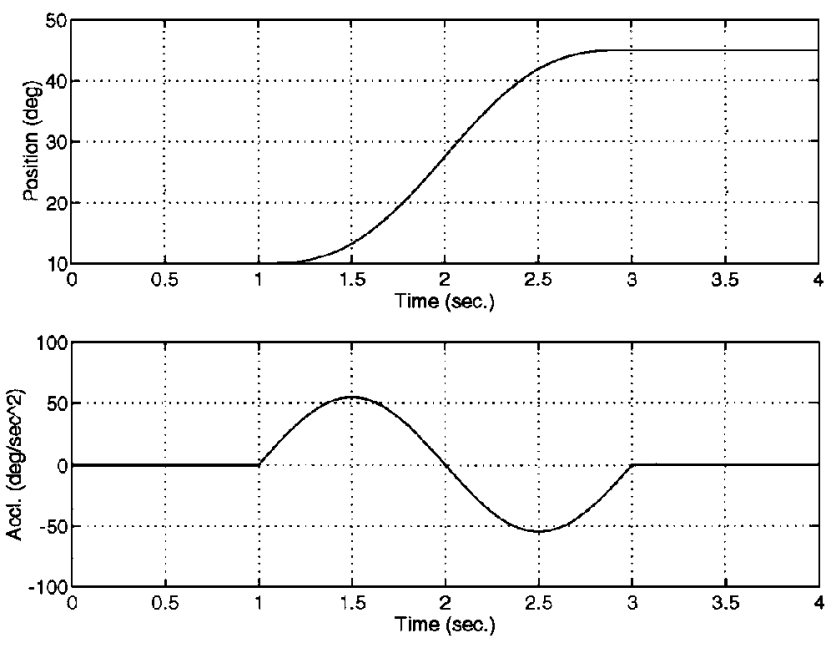

Fig. 2 Desired tip trajectory profiles for link two.

$$
A_{3}=\left[\begin{array}{cccc}
4 e & 6 e & 0 & 0 \\
6 e & 12 e & 0 & 0 \\
0 & 0 & 4 e & 6 e \\
0 & 0 & 6 e & 12 e
\end{array}\right]
$$

and $A_{2}=\alpha A_{3}$. Note we have exactly

$$
\operatorname{det} A_{3}=144 e^{4}>0
$$

$$
\begin{gathered}
\operatorname{det} A_{1}=-\frac{1}{13,230,000} m^{4}-\frac{13}{10,584,000} m^{3} m_{e} \\
-\frac{13}{5,292,000} m^{3} \frac{h}{l^{2}}-\frac{13}{44,100} m^{2} m_{e} \frac{h}{l^{2}}<0
\end{gathered}
$$

Equations (60) and (61) imply that the product of all eigenvalues of the system is negative. Because the total number of the eigenvalues is an even number, we conclude that there exists at least one positive real eigenvalue for the linearized zero dynamics (59). The nonminimum-phase property, thus, is verified. It is noticed from the preceding argument that the nonminimum-phase property is independent of $\alpha$, the damping ratio. Thus, even in the case when structural damping is neglected $(\alpha=0)$, the flexible manipulator system is still nonminimum phase.

To verify the hyperbolicity of the zero dynamics, we further assume that $m_{e}$ and $h=0$ for notational simplicity. First, zero eigenvalues can be easily excluded from the fact that $\operatorname{det} A_{3} \neq 0$. Next, suppose the zero dynamics has pure imaginary eigenvalues $\pm i \lambda$ with $\lambda \neq 0$. Substituting them into the characteristic equation of the zero dynamics leads to

$$
\operatorname{det}\left(-\lambda^{2} A_{1} \pm i \lambda A_{2}+A_{3}\right)=0
$$

Equivalently,

$$
\operatorname{det}\left(-\lambda^{2} A_{1} A_{3}^{-1}+[1 \pm i \alpha \lambda] I\right)=0
$$

Equation (62) says that there exists eigenvalue $\bar{\lambda}$ of matrix $A_{1} A_{3}^{-1}$ such that

$$
-\lambda^{2} \bar{\lambda}+[1 \pm i \alpha \lambda]=0, \quad \bar{\lambda}=\left(1 / \lambda^{2}\right)[1 \pm i \alpha \lambda]
$$

But the characteristic equation of matrix $(e / m) A_{1} A_{3}{ }^{-1}$ is exactly given by

$$
s^{4}-\frac{61}{420} s^{3}-\frac{331}{2,116,800} s^{2}+\frac{127}{42,336,000} s-\frac{1}{1,905,120,000}=0
$$

It can be easily verified that this characteristic equation has all four real roots. Thus, condition (63) cannot be true when $\alpha \neq 0$. Hence, the linearized zero dynamics (59) cannot have purely imaginary 
eigenvalues $\pm i \lambda$. However, no conclusion can be made about the hyperbolicity of the zero dynamics when structural damping is neglected $(\alpha=0)$.

The well-defined relative degree property can be seen by arranging the forward dynamics as follows. Substituting Eq. (48) into forward dynamics equation (46) yields

$$
\begin{gathered}
{\left[M_{22}(\psi)-M_{21}(\psi) D_{2}\right] \ddot{\boldsymbol{q}}+H_{2}(\boldsymbol{\psi}, \dot{\boldsymbol{\psi}})} \\
+M_{2} \dot{\boldsymbol{q}}+M_{3} \boldsymbol{q}+M_{21}(\boldsymbol{\psi}) \ddot{\boldsymbol{y}}=0
\end{gathered}
$$

It can be easily seen that matrix $M_{22}(\psi)-M_{21}(\psi) D_{2}$ evaluated at $\theta_{2}=90 \mathrm{deg}$ is exactly the matrix $A_{1}$ in Eq. (59) because Eq. (64) with $\boldsymbol{y} \equiv \boldsymbol{y}_{d}$ is the reference dynamics (49). Equation (61) says that this matrix is nonsingular. Thus, substituting Eqs. (48) and (64) into another part of the forward dynamics (45) gives

$$
\begin{aligned}
& M_{r} \ddot{\boldsymbol{y}}+H_{1}-\left[M_{12}-M_{11} D_{2}\right]\left[M_{22}-M_{21} D_{2}\right]^{-1} \\
& \quad \times\left[H_{2}+M_{2} \dot{\boldsymbol{q}}+M_{3} \boldsymbol{q}\right]=\boldsymbol{u}
\end{aligned}
$$

where

$$
M_{r}=M_{11}-\left[M_{12}-M_{11} D_{2}\right]\left[M_{22}-M_{21} D_{2}\right]^{-1} M_{21}
$$

It can be easily verified that $M_{r}$, the coefficient matrix of $\ddot{y}$, is invertible under the same assumptions as made in the verification of the hyperbolicity, thus verifying the existence of a locally welldefined relative degree. Both output components have relative degree two at the equilibrium point $\boldsymbol{\theta}=[0,90]^{T} \mathrm{deg}$. It can further be verified that the preceding argument is still valid over the range of $5 \leq \theta_{2} \leq 90 \mathrm{deg}$. The range is selected such that it covers the reference trajectory chosen in the following simulation study.

\section{Controller Structure}

The controller structure of our stable inversion method is shown in Fig. 3. From the stable inverse dynamics with specified boundary conditions, we compute the desired state trajectory $\boldsymbol{x}_{d}$ and the nominal control input $\boldsymbol{u}_{d}$. The feedback stabilizing signal $\boldsymbol{u}_{s}$ is superimposed on the feedforward $\boldsymbol{u}_{d}$ to obtain the total control input to drive the manipulator. Because the flexible modes of the arm are not measurable, the controller uses only the rigid-angle measurement for feedback, which could be easily read from encoders installed on both joints.

The first column of Table 2 lists the eigenvalues of the forward dynamics. They are computed from the linearization of forward dynamics (38) at $\boldsymbol{\theta}=[0,90]$ deg and $\boldsymbol{q}=\mathbf{0}$. The open loop is unstable because there are four poles at the origin. Therefore, a stabilizer is

Table 2 Eigenvalues of the forward dynamics

\begin{tabular}{lc}
\hline \hline Open loop & Closed loop \\
\hline 0 & $-0.2608 \pm i 0.6814$ \\
0 & $-0.9070 \pm i 11.6319$ \\
0 & $-1.4119 \pm i 1.0185$ \\
0 & $-1.7529 \pm i 5.8291$ \\
$-0.2219 \pm i 0.6581$ & $-16.3544 \pm i 54.6808$ \\
$-0.6345 \pm i 11.2469$ & $-43.8221 \pm 81.2408$ \\
$-16.2886 \pm i 4.7029$ & \\
$-42.6746 \pm i 81.9378$ & \\
\hline \hline
\end{tabular}

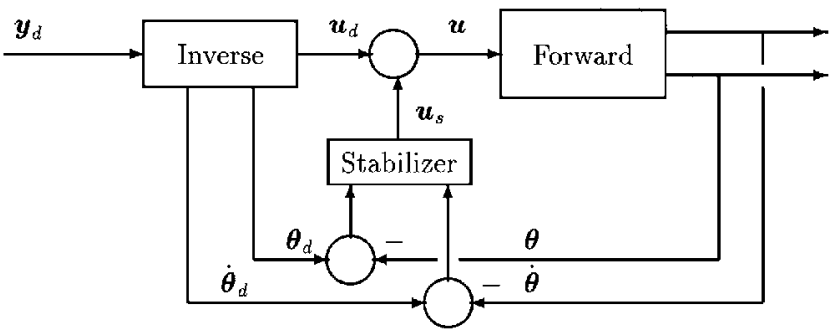

Fig. 3 Controller scheme of stable inversion. necessary. However, the topic of general nonlinear stabilization is out of the scope of this paper. In this study, a simple linear joint-angle feedback control is implemented:

$$
\boldsymbol{u}_{s}=K_{p}\left[\boldsymbol{\theta}_{d}-\boldsymbol{\theta}\right]+K_{d}\left[\dot{\boldsymbol{\theta}}_{d}-\dot{\boldsymbol{\theta}}\right]
$$

where

$$
K_{p}=K_{d}=\left[\begin{array}{cc}
0.5 & 0 \\
0 & 0.375
\end{array}\right]
$$

The gain matrices are selected to stabilize the two linearizations of the forward dynamics at $t_{0}$ and $t_{f}$. The eigenvalues of the linearization of the forward dynamics (38) at $\boldsymbol{\theta}=[0,90]$ deg and $\boldsymbol{q}=\mathbf{0}$ after stabilization are given in the second column of Table 2 . The overall feedforward plus feedback control law, thus, has the following form:

$$
\boldsymbol{u}=\boldsymbol{u}_{d}+\boldsymbol{u}_{s}=\boldsymbol{u}_{d}+K_{p}\left[\boldsymbol{\theta}_{d}-\boldsymbol{\theta}\right]+K_{d}\left[\dot{\boldsymbol{\theta}}_{d}-\dot{\boldsymbol{\theta}}\right]
$$

See Refs. 23 and 24 for more analysis on stabilization and tracking performance of controllers using stable inversion.

\section{Simulation Results}

In this subsection, we present the digital simulation results and study the performance of our stable-inversion-based tracking controller. The iterative numerical procedure discussed in Sec. II is used. For the given reference trajectory, the nominal control input $\boldsymbol{u}_{d}$ and the desired joint-angle trajectory $\boldsymbol{\theta}_{d}$ are calculated through the following steps.

1) Set $\boldsymbol{q}_{0}(t)=\mathbf{0}$ for all $t$.

2) Linearize Eq. (49) along $\boldsymbol{q}_{0}(t)$ and $\dot{\boldsymbol{q}}_{0}(t)$ to get Eqs. (22-25), (28-34), and (57).

3) Integrate Eq. (32) backward in time to get $S(t)$.

4) Integrate Eq. (33) backward in time to get $\boldsymbol{v}(t)$.

5) Integrate Eq. (34) forward in time to get $\zeta_{1}(t)$ and get $\zeta_{2}(t)$ by Eq. (30).

6) Compute

$$
\boldsymbol{q}=\left[\begin{array}{l}
C_{s} \\
C_{u}
\end{array}\right]^{-1}\left[\begin{array}{l}
\boldsymbol{\zeta}_{1} \\
\boldsymbol{\zeta}_{2}
\end{array}\right]
$$

7) If $\left\|\boldsymbol{q}-\boldsymbol{q}_{0}\right\|$ is greater than a given threshold, set $\boldsymbol{q}_{0}=\boldsymbol{q}$ and go to step 2; otherwise go to step 8 .

8) Compute the nominal input $\boldsymbol{u}_{d}$ from Eq. (50) and desired rigid mode $\boldsymbol{\theta}_{d}$ from Eq. (48).

The numerical procedure stops when it leads to a relative error of $0.0005 \%$ in $\boldsymbol{q}$ between the third and the fourth iterations. It takes a few minutes on a DEC workstation with the algorithm coded in Matlab. Figure 4 shows the joint torque $\boldsymbol{u}_{d}$ needed to produce the desired tip trajectories. As expected, the torque needs to be applied to preshape the links some time before the tip starts moving due to the nonminimum-phase property of the system.

Using the control law (66), computer simulation of the closedloop dynamics is carried out in Matlab. Figure 5 shows that the tips follow the desired trajectories exactly without any undershoot or overshoot.

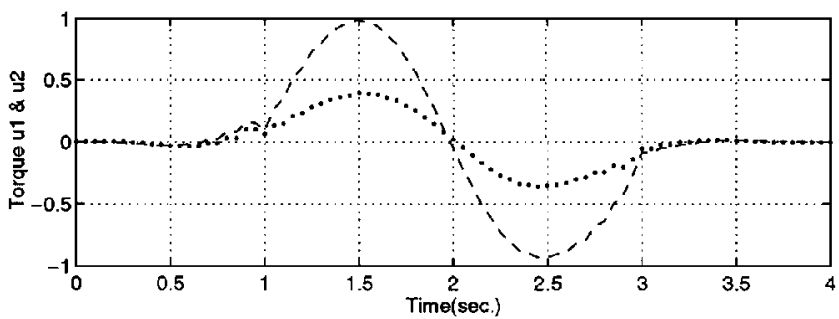

Fig. 4 Nominal control input by stable inversion. 

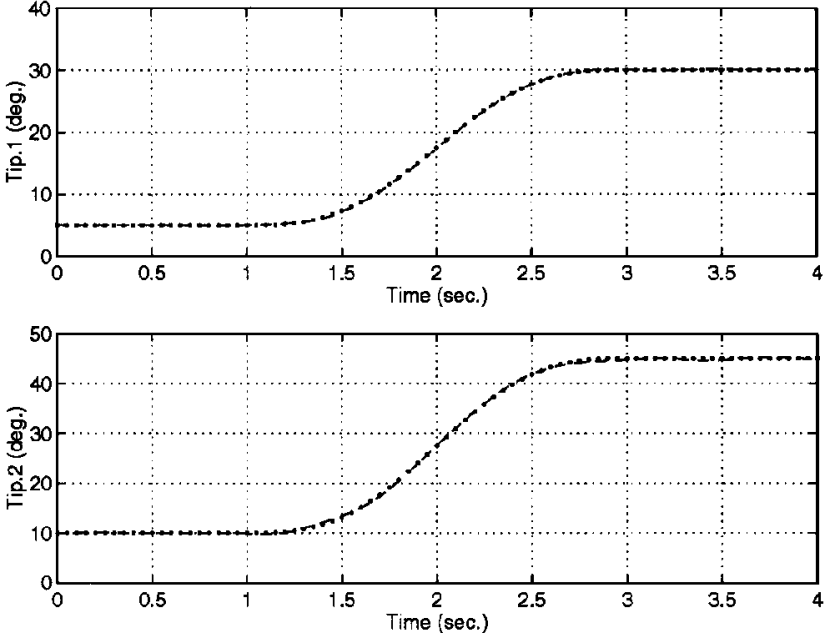

Fig. 5 Reference trajectory and trajectory by stable inversion.

\section{Conclusion}

Stable inversion of nonlinear nonminimum-phase systems is successfully applied to the tip trajectory tracking for a two-link flexible robot manipulator. Simulation results demonstrate that the stable inversion approach is very effective for obtaining stable and remarkably accurate output tracking for flexible manipulators. This approach is expected to perform equivalently well for other realistic nonminimum-phase nonlinear systems.

It is noticed that stable-inversion-basedoutput tracking uses the same controller structure of feedforward plus feedback as that used by nonlinear regulation. Hence, the approach is also a model-based approach and has the same robustness issues as nonlinear regulation does. To handle the model uncertainty, either a robust feedback controller needs to be designed or some robust stable inverse solutions be computed. The robustness issue of inverse solutions is an issue of the stable inversion theory itself and is currently under study.

\section{Acknowledgment}

This work is partially supported by the National Science Foundation under Grant ECS-9410646.

\section{References}

${ }^{1}$ Fu, K. S., Gonzalez, R. C., and Lee, C. S. G., Robotics: Control, Sensing, Vision, and Intelligence, McGraw-Hill, New York, 1987.

${ }^{2}$ Kelkar, A., and Joshi, S., Control of NonlinearMultibody Flexible Space Structures, Springer, New York, 1996.

${ }^{3}$ Cannon, R. H., and Schmitz, E., "Initial Experimental on the End Point Control of a Flexible One-Link Robot," International Journal of Robotics Research, Vol. 3, No. 3, 1984, pp. 62-75.

${ }^{4}$ Siciliano, B., and Book, W. J., "A SingularPerturbation Approach to Control Lightweight Flexible Manipulators," International Journal of Robotics Research, Vol. 7, No. 4, 1988, pp. 79-90.

${ }^{5}$ Bayo, E., "A Finite-Element Approach to Control the End-Point Motion of a Single-Link Flexible Robot," Journal of Robotic Systems, Vol. 4, No. 1 , 1987, pp. 63-75

${ }^{6}$ Kwon, D. S., and Book, W. J., "An Inverse Dynamic Method Yielding Flexible Manipulator State Trajectories," Proceedings of the 1990 American Control Conference, Vol. 1, 1990, pp. 186-193.

${ }^{7}$ Lucibello, P., and Di Beneketto, M. D., Diagnostics, Vehicle Dynamics and Special Topics, American Society of Mechanical Engineers, New York, 1989.

${ }^{8}$ Isidori, A., and Byrnes, C. I., "Output Regulation of Nonlinear Systems," IEEE Transactions on Automatic Control, Vol. 35, No. 2, 1990, pp. 131-140.

${ }^{9}$ De Luca, A., Lanari, L., and Ülivi, G., New Trends in Systems Theory, Springer-Verlag, New York, 1991.

${ }^{10}$ Brockett, R. W., and Mesarovic, M. D., "The Reproducibility of Multivariable Systems," Journal of Mathematical Analysis and Applications, Vol. 11, No. 3, 1965, pp. 548-563.

${ }^{11}$ Silverman, L. M., "Inversion of Multivariable Linear Systems," IEEE Transactions on Automatic Control, Vol. 14, No. 3, 1969, pp. 270-276.

${ }^{12}$ Sain, M. K., and Massey, J. L., "Invertibility of Linear Time-Invariant Dynamical Systems," IEEE Transactions on Automatic Control, Vol. 14, No. 2, 1969, pp. 141-149.

${ }^{13}$ Hirschorn, R. M., "Invertibility of Multivariable Nonlinear ControlSystems," IEEE Transactions on Automatic Control, Vol. 24, No. 6, 1979, pp $855-865$.

${ }^{14}$ Singh, S. N., "A Modified Algorithm for Invertibility in Nonlinear Systems," IEEE Transactions on Automatic Control, Vol. 26, No. 2, 1981, pp. $595-599$.

${ }^{15}$ Zhao, H., and Chen, D., "Exact and Stable Tip Trajectory Tracking for Multi-Link Flexible Manipulators," Proceedings of the 32nd IEEE Conference on Decision and Control, Vol. 2, Inst. of Electrical and Electronics Engineers, 1993, pp. 1371-1376.

${ }^{16}$ Chen, D., and Paden, B., "Stable Inversion of Nonlinear Nonminimum Phase Systems," International Journal of Control, Vol. 64, No. 1, 1996, pp. 81-97.

${ }^{17}$ Ortega, R., and Spong, M. W., "Adaptive Motion Control of Rigid Robots: A Tutorial," Proceedings of the 27th International Conference on Decision and Control, Vol. 2, 1988, pp. 1575-1584.

${ }^{18}$ Bayo, E., Papadopoulos, P., and Stubbe, J., "Inverse Dynamics and Kinematics of Multi-Link Elastic Robots: An Iterative Frequency Domain Approach," International Journal of Robotics Research, Vol. 8, No. 6, 1989, pp. 49-62.

${ }^{19}$ Book, W. J., Maizza-Neto, O., and Whitney, D. E., "Feedback Control of Two-Beam, Two-Joint Systems with Distributed Flexibility," Journal of Dynamic Systems, Measurement and Control, Vol. 94, No. 4, 1975, pp. 424431.

${ }^{20}$ De Luca, A., and Siciliano, B., "Inversion-Based Nonlinear Control of Robot Arms with Flexible Links," Journal of Guidance, Control, and Dynamics, Vol. 16, No. 6, 1993, pp. 1169-1176.

${ }^{21}$ Madharan, S. K., and Singh, S. N., "Inverse Trajectory Control and Zero Dynamics Sensitivity of an Elastic Manipulator," Proceedings of the 1991 American Control Conference, Vol. 2, 1991, pp. 1879-1884.

${ }^{22}$ Bayo, E., and Paden, B., "On Trajectory Generation for Flexible Robots," Journal of Robotic Systems, Vol. 4, No. 2, 1987, pp. 229-235.

${ }^{23}$ Chen, D., "Output Tracking Control of Nonlinear Nonminimum Phase Systems," Proceedings of the 33rd IEEE Conference on Decision and Control, Vol. 3, Inst. of Electrical and Electronics Engineers, 1994, pp. 23402345.

${ }^{24}$ Devasia, S., and Paden, B., "Exact Output Tracking for Nonlinear TimeVarying Systems," Proceedings of the 33rd IEEE Conference on Decision and Control, Vol. 3, Inst. of Electrical and Electronics Engineers, 1994, pp. 2346-2355. 